\title{
A toolbox for intensity modulated radiation therapy optimization
}

\author{
D. M. Shepard, Z. Jiang, and M. A. Earl \\ Department of Radiation Oncology, University of Maryland School of Medicine, Baltimore, Maryland 21201 \\ M. C. Ferris and J. Lim \\ Computer Sciences Department, University of Wisconsin, Madison, Wisconsin \\ S. Naqvi \\ Department of Radiation Oncology, University of Maryland School of Medicine, Baltimore, Maryland 21201
}

(Received 1 April 2003; revised 20 May 2003; accepted for publication 17 June 2003; published 21 August 2003)

We have designed a toolbox that provides an environment for testing radiotherapy optimization techniques, objective functions, and constraints. A set of three-dimensional (3D) pencil beam dose distributions have been computed for a cylindrical phantom. The $6 \mathrm{MV}$ pencil beams were computed using a superposition-based dose engine commissioned for an Elekta SL20 linear accelerator. Due to the cylindrical symmetry of the phantom, the pencil beam dose distributions for any arbitrary beam angle can be determined by simply rotating the pencil beam data sets. Thus, the full accuracy is maintained without the need for additional dose calculations or large data storage requirements. In addition to the pencil beam data sets, tools are included for (1) rotating the pencil beams, (2) calculating the beam's eye view, (3) drawing structures, (4) writing the pencil beam dose data out to the optimizer, and (5) visualizing the optimized results. The pencil beam data sets and the corresponding tools are available for download at http://medschool.umaryland.edu/departments/ radiationoncology/pencilbeam/. With this toolbox, researchers will have the ability to rapidly test new optimization techniques and formulations for intensity modulated radiation therapy and 3D conformal radiotherapy. (C) 2003 American Association of Physicists in Medicine.

[DOI: $10.1118 / 1.1598171]$

Key words: IMRT, optimization, pencil beam, toolbox

\section{INTRODUCTION}

For both intensity modulated radiation therapy (IMRT) and three-dimensional (3D) conformal radiation therapy, inverse treatment planning can lead to increased planning efficiency and improved treatment plan quality. The ability to test a new approach to inverse planning, however, rests on one's access to (1) patient CT data, (2) an algorithm capable of performing pencil beam dose calculations, (3) a means to write out the dose data, and (4) a tool for visualizing the optimized result. Researchers are typically unable to access the full compliment of these capabilities through commercial treatment planning systems. It therefore becomes necessary to develop a variety of in-house tools before any novel optimization research can be performed.

To facilitate future research in inverse treatment planning, we have produced a set of three-dimensional pencil-beam dose distributions computed for a cylindrical phantom. These data sets are now available through our web page. In addition, we provide tools for (1) rotating the pencil beams, (2) calculating the beam's eye view, (3) drawing structures, (4) writing the pencil beams out to the optimizer, and (5) visualizing the optimized results.

The test environment provided by this toolbox should allow for the rapid development of new inverse planning techniques. Using an accurate dose calculation, one can demonstrate the validity and capabilities of a new planning approach for a variety of target sizes and shapes.

This toolbox is an extension of previous work performed at the University of Wisconsin involving the use of 2D, nondiverging, monoenergetic pencil beams. ${ }^{1}$ By contrast, the current data set includes 3D pencil beams commissioned to match a $6 \mathrm{MV}$ beam from a linear accelerator. This is of critical importance, because many optimization techniques do not scale well to 3D. Also, some of the inverse planning problems encountered in radiation therapy (such as the direct optimization of aperture shapes) are inherently 3D in nature.

\section{MATERIALS AND METHODS}

The pencil beams are incident on a water-equivalent cylinder $20 \mathrm{~cm}$ in diameter and $16 \mathrm{~cm}$ long. The centering of the phantom coincides with the machine isocenter which is 100 $\mathrm{cm}$ from the source. Pencil beams of $1 \mathrm{~cm} \times 0.5 \mathrm{~cm}$ size were calculated for a $20 \times 20$ field incident from a single direction (gantry $=0$ degrees). Consequently, there are 800 pencil beams. Each pencil beam has an associated dose matrix that is $125 \times 125$ by 31 in size.

The cylindrical symmetry of the geometry offers a significant computational advantage: the pencil beams for any gantry angle can be obtained by simply rotating the pencil beams computed for a gantry angle of zero. This rotation can be performed using the tools provided in the package. The accuracy of the pencil beam calculation is thus maintained without the need to recompute the dose for each specific case.

Sample targets and sensitive structures are included with the toolbox. In addition, a tool is provided for drawing struc- 


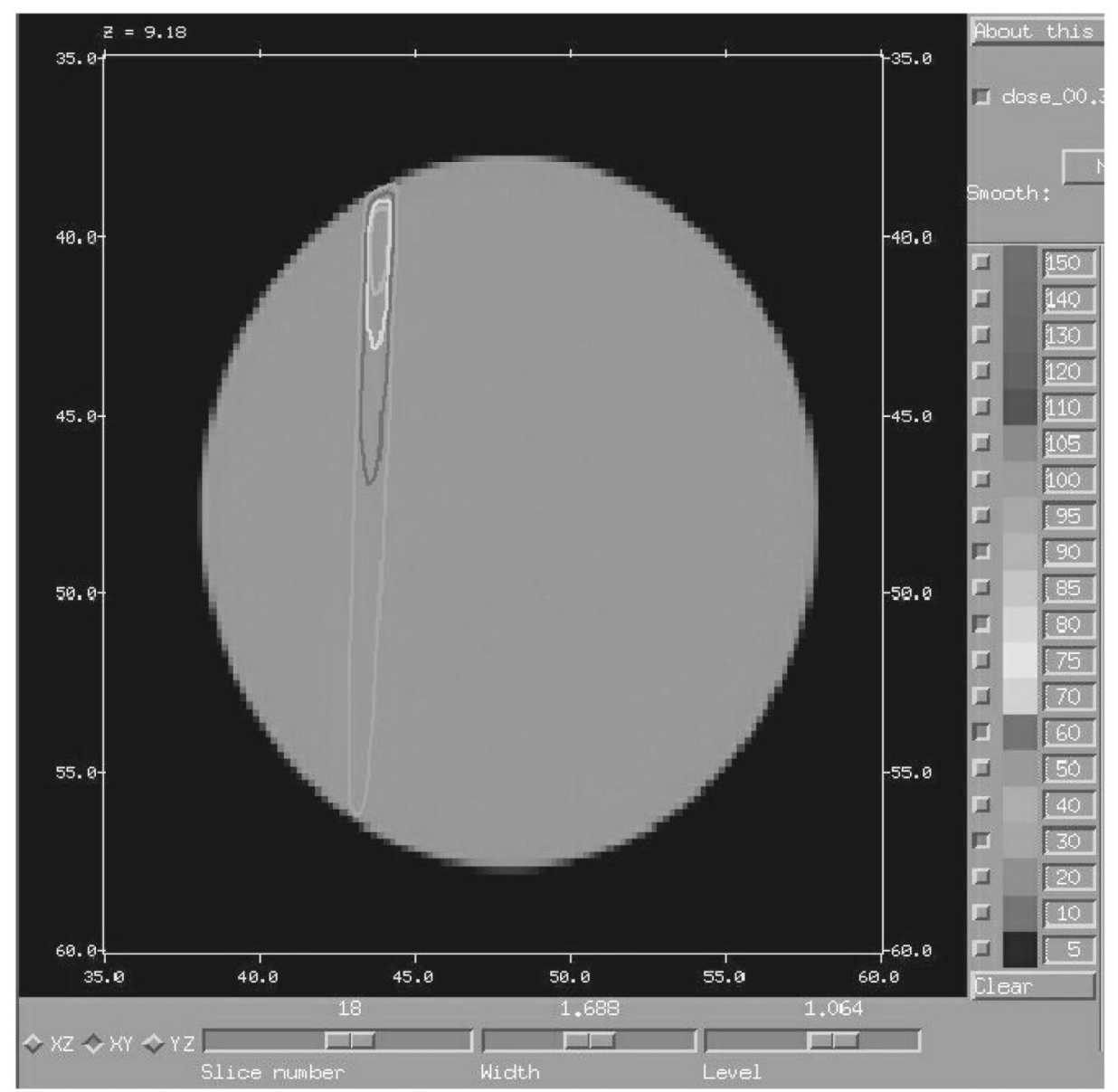

FIG. 1. A pencil beam dose distribution.

tures and generating the beams's eye view. This allows the user to test targets of various sizes and shapes. With the beam's eye view (BEV) tool, one can choose to exclude pencil beams outside of the BEV for the purpose of IMRT optimization. Also, one can choose to add together all of the pencil beams within the $\mathrm{BEV}$ to create a $3 \mathrm{D}$ conformal beam.

The pencil beams were calculated using our in-house dose

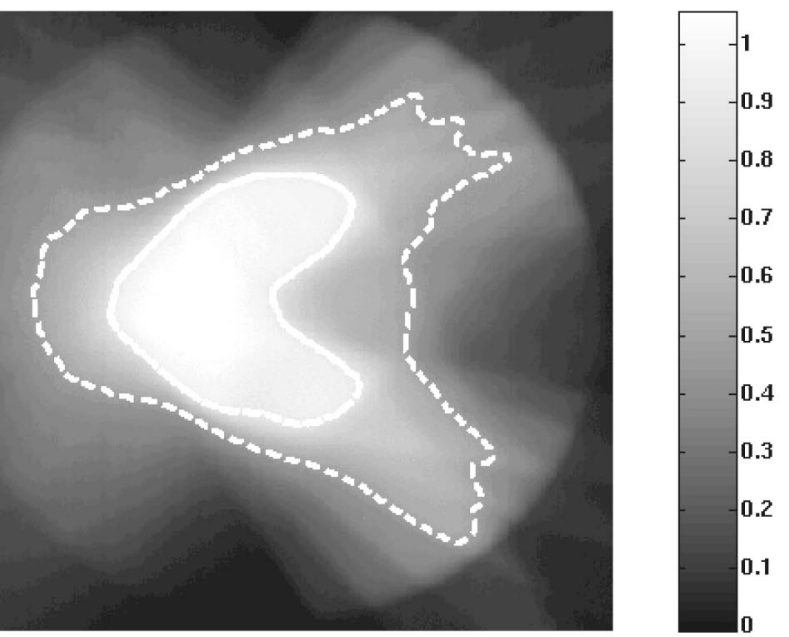

b

FIG. 2. (a) An optimized IMAT treatment plan for a c-shaped target. (b) The delivered dose distribution as measured using film. In both cases, the $90 \%$ and $50 \%$ isodose lines are plotted as white dashed lines. 
engine that combines aspects of Monte Carlo with those of the convolution-superposition technique. ${ }^{2}$ Individual photons are tracked by sampling an interaction point and then propagating the point dose kernel from that point. The model was tuned to match a $6 \mathrm{MV}$ beam from an Elekta SL20 linear accelerator.

The toolbox is designed to run on a LINUX platform. The toolbox includes the source code (written in $\mathrm{C}$ ) for reading in the pencil beams, rotating the pencil beams, determining the beam's eye view, and writing out the data for the optimizer. With modifications to the code, it is possible to write out the data in any desired format. This capability should make it possible to interface to any existing optimization code. The tools for drawing structures and visualizing the dose are provided in an executable form, and the pencil beam data are stored in a binary format.

\section{RESULTS}

Figure 1 shows the dose distribution for two adjacent pencil beams added together to create a single pencil beam $1 \mathrm{~cm}$ in width. We have used this toolbox extensively in the development of inverse planning techniques for step-and-shoot IMRT, intensity modulated arc therapy (IMAT), and threedimensional conformal therapy. ${ }^{3-5}$ Figure 2(a) shows an isodose plot for an optimized IMAT plan using six arcs. This plan was delivered on an Elekta SL20 linear accelerator, and the results from a film measurement are plotted in Fig. 2(b).
In this case, the optimization was performed using a technique called direct aperture optimization (DAO) described elsewhere. ${ }^{3}$

\section{CONCLUSIONS}

A pencil beam toolbox has been developed that allows for the rapid development and testing of radiotherapy optimization techniques. The data set and associated tool are available for download at http://medschool.umaryland.edu/ departments/radiationoncology/pencilbeam/

\section{ACKNOWLEDGMENT}

This work is supported by the National Science Foundation under Grant No. ACI-0113051.

${ }^{1}$ D.M. Shepard, G.H. Olivera, L. Angelos, O.A. Sauer, P. Reckwerdt, and T.R. Mackie, "A simple model for examining issues in radiotherapy optimization,” Med. Phys. 26, 1212-1221 (1999).

${ }^{2}$ S. Naqvi et al., Convolution superposition using the Monte Carlo method (unpublished).

${ }^{3}$ D.M. Shepard, M.A. Earl, S. Naqvi, and C. Yu, "Direct aperture optimization: A turnkey solution for step-and-shoot IMRT," Med. Phys. 29, 1007-1018 (2002).

${ }^{4}$ M.A. Earl, D.M. Shepard, S. Naqvi, X.A. Li, and C.X. Yu, "Inverse planning for intensity modulated arc therapy (IMAT) via direct aperture optimization," Phys. Med. Biol. 48, 1075-1089 (2003).

${ }^{5}$ J.H. Lim, M.C. Ferris, and D.M. Shepard, An Optimization Framework for Conformal Radiation Treatment Planning, Optimization Technical Report 03-01, University of Wisconsin, 2003. 